\title{
Modeling topologically resilient supply chain networks
}

\author{
Viplove Arora (D) and Mario Ventresca*
}

\author{
${ }^{*}$ Correspondence: \\ mverntresca@purdue.edu \\ School of Industrial Engineering, \\ Purdue University, 315 N. Grant \\ Street, 47907-2023 West Lafayette, \\ IN, USA
}

\begin{abstract}
The ubiquity of supply chains along with their increasingly interconnected structure has ignited interest in studying supply chain networks through the lens of complex adaptive systems. A particularly important characteristic of supply chains is the desirable goal of sustaining their operation when exposed to unexpected perturbations. Applied network science methods can be used to analyze topological properties of supply chains and propose models for their growth. Network models focusing on the critical aspect of supply chain resilience may provide insights into the design of supply networks that may quickly recover from disruptions. This is vital for understanding both static and dynamic structures of complex supply networks, and enabling management to make informed decisions and prioritizing particular operations. This paper proposes an action-based perspective for creating a compact probabilistic model for a given real-world supply network. The action-based model consists of a set of rules (actions) that a firm may use to connect with other firms, such that the synthesized networks are topologically resilient. Additionally, it captures the heterogeneous roles of different firms by incorporating domain specific constraints. Results analyzing the resilience of networks subjected to node disruptions show that networks synthesized using the proposed model can generally outperform its real-world counterpart.
\end{abstract}

Keywords: Network modeling, Supply chains, Topological resilience

\section{Introduction}

Present day supply chain networks (SCNs) are profoundly interconnected structures that emerge from a largely downstream exchange of goods between firms (manufacturers, distributors, retailers, etc.) that are involved in creating a set of final products. Connections are formed or removed as firms use information from a local neighborhood to increase the value they derive from the supply chain without any knowledge of the interconnection structure of the whole supply network. That is, the network itself emerges through the local decisions of firms (Brintrup et al. 2016). Despite this realization, most industrial operations are still built upon overly simplified (often highly linear) models (Brintrup et al. 2015). Other gaps were identified in (Bellamy and Basole 2013) that suggest a focus on supply chain structure, dynamics and design strategy. Subsequently, there might be a tremendous unlocked potential in supply network efficiency that can be achieved through a complex systems/networks perspective (Choi et al. 2001; Surana et al. 2005). Additionally, three other major challenges have been identified as critical to the study of supply

(c) The Author(s). 2018 Open Access This article is distributed under the terms of the Creative Commons Attribution 4.0 International License (http://creativecommons.org/licenses/by/4.0/), which permits unrestricted use, distribution, and reproduction in any medium, provided you give appropriate credit to the original author(s) and the source, provide a link to the Creative Commons license, and indicate if changes were made. 
networks through the lens of complex networks (Pathak et al. 2007): (i) Researchers' ability to comprehend the complex interactive nature of supply chain formation is limited, especially as the network size increases; (ii) Effective metrics for the dynamic nature of supply chain evolution are lacking; and (iii) developing theories to support supply chain design principles in the presence of network adaptation is nontrivial.

Utilizing the knowledge of network science to study supply chain networks was first suggested in (Choi et al. 2001), where various recommendations for future research directions on bridging the two research areas were laid out. Subsequent examinations of supply chain networks through the lens of network science have primarily focused on analyzing topological characteristics of supply chains and providing summary statistics for describing particular features. This is particularly useful since analysis of topological characteristics of the interconnection structure of firms in a supply chain enables managers to reflect on various aspects of the supply chain. For instance, (Kim et al. 2011; Borgatti and Li 2009) investigated automobile manufacturer supply networks with the aim of understanding the implications of using well known social network measures in the context of supply chains. Similarly, (Lomi and Pattison 2006) examined the network of automotive firms in southern Italy and discovered high local clustering, while (Keqiang et al. 2008) had very similar observations for the Guangzhou automotive network. Though an assortment of comparative investigations have been performed in other industry settings revealing fascinating properties of the networks themselves, consensus on a standard approach for designing supply networks remains generally elusive (Bellamy and Basole 2013). Though these surveys of real-world supply networks and their reported summary statistics provide insights into predominant characteristics of supply chains, they provide limited insight into the mechanisms by which these networks grow and evolve. A major reason for this stagnation has been a lack of availability of real-world supply network data to study, leading to a significant need for generators (algorithms for creating networks with specific topological properties) capable of synthesizing realistic supply networks that can be utilized to derive deeper insight into their best design principles. The ability of a network generator to synthesize networks with similar underlying summary statistics can help us understand the result of natural and deliberate perturbations on the overall functionality of the supply chain.

Network generators provide a platform for deriving deeper insights into complex networks and the processes that give rise to their observed structures. Their origins can be traced to random graphs (Erdös and Rényi 1959), which assume a constant number of nodes and edge existence with uniform probability. Random graphs are unlikely to be representative models of real-world systems, but a variety of better approaches have since been proposed that define network growth dynamics and non-uniform edge existence (see (Fienberg 2012; Goldenberg et al. 2010; Barthélemy 2011; Newman 2003) for a more comprehensive overview). Other popular network generators include the smallwold model (Watts and Strogatz 1998) (i.e. a network in which nodes can reach other nodes in a small number of steps), and scale-free model (Barabási and Albert 1999) (i.e., the probability $P(k) \approx k^{-\alpha}$, for degree $k$ with usually $\left.\alpha \in(2,3)\right)$. One of the most popular statistical network models are the exponential random graphs (Strauss 1986; Wasserman and Pattison 1996; Anderson et al. 1999). These models deal with link formation mechanisms using conditional dependence, which states that the existence of links in a network is shaped by the presence or absence of other links (and possibly node-level attributes) 
(Robins et al. 2007). These models have limited utility for synthesizing supply chain networks due to the absence of mechanisms to incorporate real-world supply chain constraints. These methods have been shown to be highly unlikely to synthesize networks that share a strong structural resemblance to actual supply networks and are woefully insufficient to study the intricate nature of supply chains, therefore being unsuitable for discovering new design principles (Bellamy and Basole 2013; Kim et al. 2011). Incorporating constraints on nodes (i.e., firms) is generally outside the capabilities of most existing network generators, which is why most existing studies have concentrated on general analyses with limited insight into new design principles (Bellamy and Basole 2013).

A particularly important characteristic of dynamic supply chains is the desirable goal of sustaining their operation when exposed to unexpected disruptions. The goal of supply chain robustness is to sustain operation during such disruptions, whereas the goal of supply chain resilience focuses on designing systems that quickly recover from these disruptions. (Perera et al. 2015) suggests that the definition of resilience and robustness should be established in parallel with the definition of disruption, and shows how some of the important research in supply chains have accomplished this. These terms are often used interchangeably in the literature, but in either case the impact may be significant. For instance, according to a 2017 report by the Business Continuity Institute (Alcantara et al. 2017) 75\% of businesses experience at least one supply chain disruption every 12 months (although they suggest the value is likely higher due to underreporting), $22 \%$ report cumulative losses of at least $\$ 1$ million over this time, with $34 \%$ reporting at least $\$ 270,000$. Additionally, $55 \%$ reported a loss in productivity, $34 \%$ reported that their service was impaired, and 32\% reported a loss in revenue. These trends have led to a shift in focus of research from supply chain efficiency to supply chain resilience (Perera et al. 2015).

(Klibi et al. 2010) suggested that topological resilience should be assessed when designing supply chains to ensure sustainable value creation. Robustness and resilience have thus become important areas of study (for simplicity we refer to both as resilience). While (Thadakamalla et al. 2004) was the first to use topology of SCNs for studying resilience, subsequent papers like (Zhao et al. 2011b; Wang et al. 2015) provide supply chain design insights by examining resilience against both random and targeted attacks. Numerous specialized measures of resilience have also been proposed for supply chains (Barroso et al. 2015), but most analyses concentrate on empirical studies from a centralized context. Outside of the supply chain network community, resilience has also attracted significant attention (see for example (Gao et al. 2016)). A resilient supply chain should rapidly and effectively respond to perturbations such as supply or demand fluctuations, or to complete or partial failure of a subset of firms. However, being a complex system, adaptation to changes in the supply chain cannot be dictated by those overseeing or relying upon it. Instead, structural resilience should exist as an outcome of the local linking decisions of various firms within a supply chain without explicit awareness of the overall structure.

A significant amount of research examining complex network models for supply chain networks has focused on using straightforward and conventional strategies as models for SCN evolution. To understand these network models, we first need to introduce two concepts that are essential ingredients of any such approach: (i) each firm belongs to a unique tier, which corresponds to its distance (number of hops in the networks) from the 
consumer in the final supply chain with a restriction that supply-demand relations occur only between firms in adjacent tiers; (ii) every firm has a fixed role in the network, i.e. it is a supplier, manufacturer, consumer etc. Most supply chain models categorize nodes based on their tiers and roles, and then use these attributes to define attachment rules, for example (Thadakamalla et al. 2004; Wang et al. 2015) use a heterogeneous preferential linking mechanism that varies based on role of the firm, (Nair and Vidal 2011) proposes an agent-based model that uses firm role and demands to form links, (Wen et al. 2012; Mari et al. 2015) use restricted preferential attachment mechanism based on firm tiers. Others use more complicated linking mechanisms such as, local selection and preferential attachment (Li et al. 2013; Zhao et al. 2011b), random, preferential attachment and similarity (Xuan et al. 2011), and fitness based attachment (Perera et al. 2017c). Though these models incorporate basic features for modeling SCNs, their simple attachment mechanisms cannot replicate the topology of real-world SCNs, as their capabilities are restricted to synthesizing networks that reproduce a few characteristics like power law degree distributions. Further, only a few of these models focus on creating resilient SCN topologies, without providing any insights into supply chain design principles. Thus, there is still a significant gap in developing a generalizable network growth model that can generate topologies mimicking real-world SCNs (Perera et al. 2017a, b).

\section{Main contributions}

In this paper, we focus on modeling of supply chain networks by utilizing the actionbased framework (Arora and Ventresca 2017) for learning a compact probabilistic model for a given material flow SCN. The proposed framework can learn a compact model using a single observation of a real-world supply network and the obtained parameters can be used to synthesize, with high probability, statistically similar networks to a given supply network. (Arora and Ventresca 2018) modified the action-based approach for the supply chain context by considering directed networks and incorporating domain specific constraints. Additionally, (Arora and Ventresca 2018) used the role of a firm (manufacturer, supplier, distributor etc.) to infer constraints and growth mechanisms for SCNs. Here instead we utilize tier information to impose linking constraints among firms, while preserving the tiered structure of the target SCN. The modified network generator that captures critical real-world constraints concerning rules by which firms exchange goods is described in "Action-based networks" section. The novel framework is then used for modeling and synthesizing 10 realistic supply networks in "Results" section. The applicability of the framework for modeling real-world supply networks is tested, and the resilience of the synthesized networks is analyzed by subjecting them to random and targeted node disruptions. The probabilistic model can also be used to infer growth mechanisms of real-world SCNs by examining the optimized parameters. Finally, "Conclusions and future work" section concludes the paper with some conclusions and directions for future research.

\section{Action-based networks}

The underlying goal of this research is to define an adequately robust procedure that can synthesize networks exhibiting structural properties observed in real-world SCNs. A network generator is considered to be synthesizing realistic networks if a topological comparison between the synthesized and real networks is, with high likelihood, 
statistically similar across a subset of user-desired topological characteristics. In this way, the objective isn't to exactly replicate the topology of a given real-world network because there is no utility in synthesizing isomorphic networks as it provides no additional insights. Further, given the input is a single SCN observation, strong assumptions about the dynamical growth of the network need to be made, and the network generator needs to be robust to any such assumptions. Finally, the parameters of the optimized network generator should ideally provide additional insights into the local decisions of the firms that might have lead to creation of the observed network topology.

The problem of discovering a network generator can be posed as a non-linear inverse problem having the form $S(\mathcal{T}) \vdash F(\mathbf{M}, \xi)$ (i.e. $F(\mathbf{M}, \xi)$ can be inferred from $S(\mathcal{T})$ ). The target network $\mathcal{T}$ and set of $p$ user-desired network structural properties $S(\mathcal{T})=$ $\left\{s_{1}(\mathcal{T}), \ldots, s_{p}(\mathcal{T})\right\}$ of interest are given as input to the system. Therefore, the goal is to infer $\mathbf{M}$ under the assumption that network formation is performed by the forward operator $F$. Here, $F$ is an algorithm capable of synthesizing networks based on a random process $\xi \in \Xi$ that can be used to obtain a finite set of networks by repeated simulation of $F(\mathbf{M}, \xi)$.

Alderson (2009) considered network generation as an optimization-based reverseengineering problem and concluded that a "good" forward model should consider both the structure and function of the network (although a procedure to accomplish the task was not given). An action-based network generator (ABNG) is proposed as the forward operator $F$, which assumes that complex networks emerge through local interaction among nodes that make linking decisions while completely ignoring the global network topology. This assumption is particularly appropriate for modeling supply chain networks because its overall structure can be understood as a self-organizing system that consists of various entities engaging in localized decision making (Perera et al. 2017a). Given a network observation, it might not be possible to uncover the exact reason(s) behind the existence of a particular link/interaction among firms, but one could potentially list a finite number of decision processes that might have lead to creation of a particular link. A probability distribution on these decision processes can then be defined that can potentially model the existence of that link with a high likelihood. For example, the exact reason for interaction between two specific firms may not be known, but potential reasons for the interaction can be enumerated and assigned a corresponding probability.

The inverse problem of identifying the probabilities when ABNG is used as the forward operator can be formulated in an optimization-based framework, where the goal is to minimize a cost function $D$ that can serve as a metric to evaluate the difference between the networks synthesized using ABNG and the given target network. Given an appropriate probabilistic model with a viable set of decision processes, it seems logical that a variety of network topologies can be synthesized. So, the fundamental idea behind action-based networks is to define a unifying network generative process, which follows from observations by (Zheng et al. 2014) who note that there must exist an assembling algorithm to combine local mechanisms for emergence of different complex network structures. Identifying such an assembling algorithm can help us distinguish between local decision processes that are responsible for network growth from those that emerge as byproducts of the network generation process.

Consider a network $\mathcal{G}=(V, E)$, where $V$ is the set of $n$ firms $\left\{v_{1}, \ldots, v_{n}\right\}$ and $E$ is the set of directed edges showing material flow between firms in $V$. Let there exist an action set 
$A=\left\{a_{1}, \ldots, a_{k}\right\}$ containing $k$ node actions, each representing a single decision process for creating an edge $\left(v_{i}, v_{j}\right)$ from a node $v_{i} \in V$ to any node $v_{j} \in V$, where $i \neq j$. Without domain specific knowledge actions for choosing $v_{j}$ can be based on network topological characteristics. For example, a set of four actions could be:

$$
A=\left\{\begin{array}{l}
a_{1}=\text { probabilistically select } v_{j} \text { based on its degree, } \\
a_{2}=\text { select a second neighbor } v_{j} \text { uniformly at random, } \\
a_{3}=\text { select } v_{j} \text { as node having highest Jaccard similarity to } v_{i}, \\
a_{4}=\text { do not make an edge. }
\end{array}\right\}
$$

Let $\mathbb{P}\left(A=a_{l}\right)$ be the probability that action $a_{l} \in A$ is chosen by a node, implying that nodes are homogeneous with respect to their probability of choosing actions to form connections. However, actors in real-world networks are heterogeneous with respect to their strategies for forming connections, thereby rendering this assumption unlikely to be true. Hence, $\mathbb{P}\left(A=a_{l}\right)$ must be conditioned on the type of node, where the probability distribution over actions sufficiently differs between any two node types, and every node is classified as being one and only one of the types. That is, let $\mathbb{P}(T=t)$ be a column vector storing the probability of a node being of type $t=1, \ldots, d \ll|V|$. An action matrix $\mathbf{M}=\left[\mathbb{P}\left(A=a_{l}\right) \mid \mathbb{P}(T=t)\right]$ can be used to define an action-based network generative process for a given set of nodes $V$. M could be user-defined, learned from multiple network observations, or optimized or estimated from even only one network observation (Arora and Ventresca 2017). For a finite set $S(\mathcal{T})$ of user-chosen network characteristics, the problem of determining $\mathbf{M}$ can be formulated as:

$$
\begin{array}{lll}
\operatorname{minimize} & \mathbb{E}[D[S(\mathcal{T}), S(F(\mathbf{M}, \xi))]] & \\
\text { subject to } & \sum_{j=1}^{k} M_{i, j}=1 & \forall i=1, \ldots, d \\
& \sum_{i=1}^{d} M_{i, k+1}=1 \\
& M_{i, j} \geq 0
\end{array} \quad \forall i=1, \ldots, d \text { and } \forall j=1, \ldots, k+1
$$

where $D[S(\mathcal{T}), S(F(\mathbf{M}, \xi))]$ is a measure to quantify the dissimilarity between a synthesized network $\mathcal{G}=F(\mathbf{M}, \xi)$ and target $\mathcal{T}$ based on network characteristics $S(\mathcal{T})$, and the $(k+1)^{t h}$ column of $\mathbf{M}$ corresponds to $\mathbb{P}(T=t)$. It must be noted that synthesizing isomorphic graphs is the trivial solution to the problem in Eq. (1) and is not desirable. A pictorial representation of this procedure can be seen in Fig. 1.

\section{Action set for SCNs}

While empirical studies have highlighted that it is highly unlikely that a real-world SCN might have evolved through a single linking mechanism, it is possible to conceive growth and design principles from the global properties of existing SCNs (Perera et al. 2017a). The action-based framework provides a platform for probabilistically aggregating various local linking mechanisms using a generative algorithm. Each action in ABNG serves as a single linking mechanism, which when combined with an appropriate synthesis algorithm $F(\cdot)$ can synthesize networks exhibiting varying topological characteristics. In the context of SCNs, an action is a decision process that a firm uses to select firms that it should supply its materials to. The supply chain literature provides a rich source for potential decision processes (Pathak et al. 2009; Xuan et al. 2011; Kim et al. 2011; Bellamy and Basole 2013; Hearnshaw and Wilson 2013; Wang et al. 2015), while providing insights 


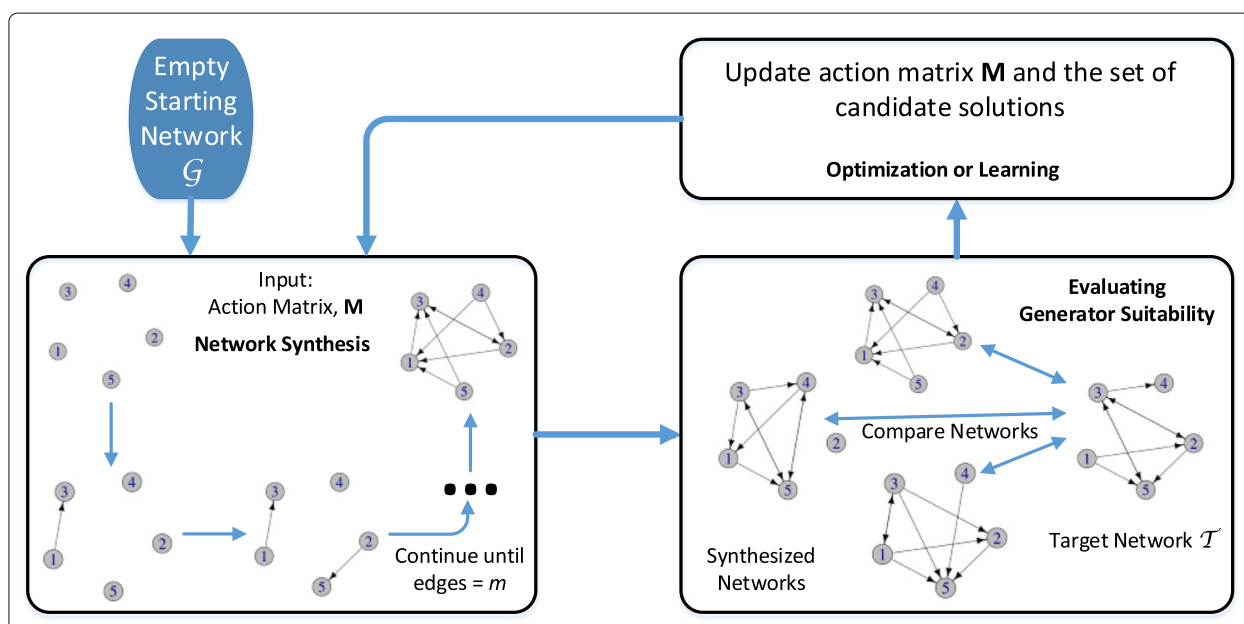

Fig. 1 A procedure for determining action matrix $\mathbf{M}$ : Network Synthesis probabilistically adds the required number of edges starting from an empty network. Evaluating Generator Suitability compares a set of synthesized networks to the target using the user-defined structural characteristics in order to determine the representativeness of action matrix $\mathbf{M}$. Finally using Optimization or Learning the action matrix $\mathbf{M}$ is perturbed, and a set of best-fit solutions are retained. The process repeats until a termination criterion (e.g., number of iterations) is satisfied

regarding how to choose a set of actions that may lead to construction of topologically resilient SCNs. The idea is to carefully choose actions for network synthesis at the micro level such that the resilience of the whole supply network gets mirrored at the macro level. The reason behind this choice is that creation of resilient structures is an expected outcome of the local linking between nodes rather than a goal of the participating firms (a firm is more likely to focus on its operational efficiency).

Recent research (Kim et al. 2015; Hearnshaw and Wilson 2013) has suggested that existence of power law degree distributions in supply chain networks has a positive affect on its resilience. Preferential attachment mechanisms have been shown to synthesize networks that perform well under random failures and are among the most prominent rules for making linking decisions, hence making them a perfect candidate for actions for SCN modeling (Thadakamalla et al. 2004; Zhao et al. 2011b; Xuan et al. 2011; Mari et al. 2015). Preferential attachment also leads to creation of networks exhibiting power law degree distributions. A variety of preferential attachment mechanisms based on network centrality metrics can potentially lead to creation of a few different hubs, hence dispersing the influential nodes across the overall supply chain. Further, networks with power law degree distributions that are formed by fractal mechanisms show greater resilience against cascading failures as compared to those obtained from the simple preferential attachment mechanisms.

Consequently, the action set $A$ will include preferentially selecting a node based on its out-degree, in-degree, vertex betweenness and closeness. These centrality metrics can induce the creation of a diverse range of hub nodes leading to creation of an overall resilient network structure. The use of network properties like betweenness and closeness for preferential linking can be seen as a proxy for more practical information such as price, performance, and quality that are more relevant in the context of supply chains (Jain et al. 2009; Li et al. 2013). A fractal mechanism based on difference in total degree (resulting in repulsion between hub nodes) has also been shown to produce resilient structures (Hearnshaw and Wilson 2013; Song et al. 2006), and is included as an action. 
It is possible that a firm does not prefer one particular firm over another based on the actions described above, leading to an action corresponding to random selection among the firms satisfying the tier constraints. An action is also based on connecting with closer nodes ${ }^{1}$ with higher probability (Zhao et al. 2011b). Finally a firm might choose to not add an edge, which is the final action in $A$. It should be noted that in the presence of no edges in the network all actions become equivalent to a random action, i.e. randomly selecting a firm satisfying the tier constraints. This is further explained along with the synthesis algorithm in "Network synthesis" section.

\section{Network synthesis}

A forward operator $F$ in case of ABNG is a generative algorithm $F(\mathbf{M}, n, m, \xi)$ that can be used to synthesize networks containing $n$ nodes and $m$ edges using an action-based model $\mathbf{M}$. The generative algorithm synthesizes networks by first creating the set of desired nodes, and then probabilistically assigning a node type $t$ to each node according to $\mathbb{P}(T=t)$. Monte Carlo simulation is then performed using the probabilities of node actions from $\mathbb{P}\left(A=a_{l} \mid t\right)$. For example, a model of a simple preferential attachment network will contain a single node type that has a sufficiently high probability for the action of creating connections to nodes based on degree. Repeated sampling from $\mathbb{P}\left(A=a_{l}\right)$ will yield a preferential attachment network, as desired. Increasingly complicated topological properties will emerge with increased number of node types and variety of actions.

Importantly, the synthesis algorithm allows the network modeler to easily integrate domain specific rules or constraints by implementing a problem specific set of node actions (e.g., ways firms could interact with each other). Moreover, the modeler may wish to ensure a specific network backbone, which can be easily accommodated by defining the initial topology before executing the Monte Carlo simulation. Termination conditions for the synthesis algorithm are user defined, e.g., certain number of edges created or topological characteristics have satisfactorily emerged.

\section{Incorporating tier constraints}

Supply chain networks are formed from heterogeneous types of nodes, where each node has a specialized task. Hence, extra care must be taken to appropriately capture critical real-world constraints concerning the rules firms use to exchange goods. Some are trivial, while others are context-dependent. Failure to reasonably accommodate these constraints in the generative process will severely limit its utility to providing only very general insights into SCN design principles. Previous research has suggested that SCNs should be modeled as tiered networks, where each tier contains nodes performing different functional tasks and the constraints of edge formation apply to the entire set of nodes in a particular tier (Bell et al. 2017, Hearnshaw and Wilson 2013, Pathak et al. 2007, 2009, Wang et al. 2015). As described in "Introduction" section, each firm in a supply chain belongs to a unique tier, which corresponds to the number of hops from the consumer in the final supply chain. For a network $\mathcal{G}=(V, E)$, the set of nodes can be partitioned into $l$ tiers $V=T_{0} \cup \cdots \cup T_{l-1}$, such that for $\alpha \neq \beta, T_{\alpha} \cap T_{\beta}=\emptyset, \forall \alpha, \beta \in\{0, \ldots, l-1\}$. Similarly, tiers also introduce constraints on the possible set of edges, such that a node $v_{i} \in T_{\alpha}$ $(\alpha \geq 1)$ can only supply materials to a node $v_{j} \in T_{\alpha-1}$, i.e. supply-demand relations exist only between firms in adjacent tiers. Algorithm 1 shows how ABNG is used to synthesize tiered supply networks for a given action matrix $\mathbf{M}$ by restricting actions to select nodes that satisfy tier constraints. 


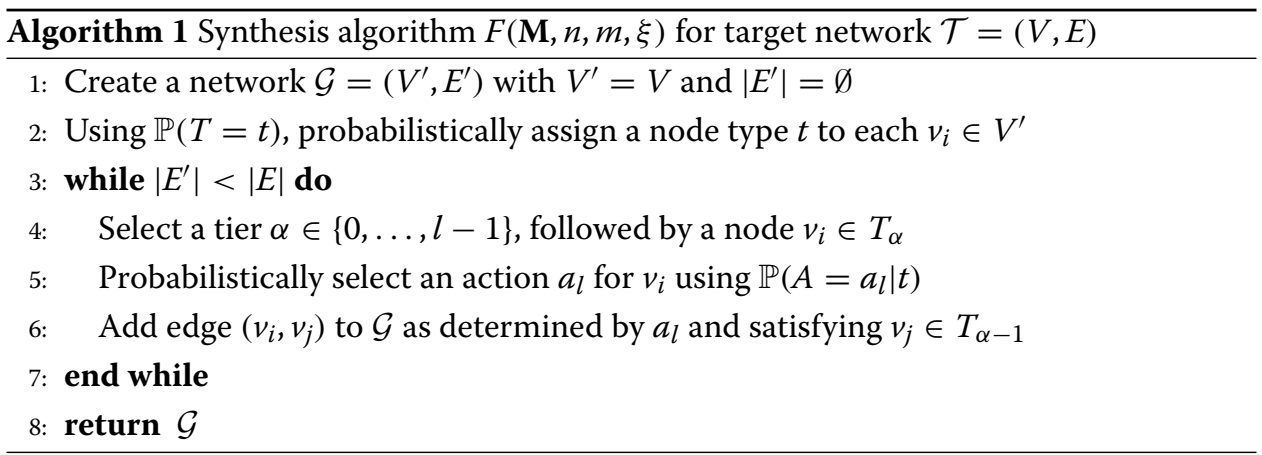

\section{Optimization and determining generator suitability}

As seen in Eq. (1), the problem of finding an action matrix $\mathbf{M}$ is framed as a multiobjective problem. The decision to frame this within a multiobjective context is based on numerous observations in network science literature arguing that it is a robust approach to determining generator suitability (Pržulj 2007; Harrison et al. 2016; Fay et al. 2014; Yaveroğlu et al. 2015). To solve this multi-objective search problem, we implement Pareto Simulated Annealing (PSA) (Czyzak and Jaszkiewicz 1998), as it is known to be a useful metaheuristic capable of global optimization in a large search space in a fixed amount of time. Additionally, only one evaluation of the objective function is required at each iteration when compared with population-based algorithms, which require an evaluation for each member of the population.

Choice of Objectives: An objective of the current research is to learn the action-based model for a given $\mathrm{SCN}$, while preserving its resilience properties. Network resilience has emerged as a critical topic in supply chain research, and a summary of several metrics that may help understand supply network resilience can be seen in (Kim et al. 2015). Recent research (Zhao et al. 2011b; Thadakamalla et al. 2004) has uncovered the importance of network topology in determining resilience of SCNs under random and targeted disruptions, hence highlighting the importance of considering it as an essential component of SCN modeling. To preserve the structural and resilience properties of the real-world SCN, the action-based framework uses the 2-sample KolmogorovSmirnov statistic to quantify difference in distribution of node level properties between the synthesized and target networks. In the current experiments, node betweenness, indegree and out-degree are utilized as the global network characteristics $S(\mathcal{T})$, but the approach is indifferent to the choice of objectives, and other properties with alternative approaches like KL-divergence or entropy-related measures can be used to quantify the difference in distributions of network properties. The choice of objectives is based on the efficacy of these measures to capture the essential structural features of a network, especially in the the context of our action-based approach (Arora and Ventresca 2017). (Kim et al. 2015) uses these network properties to understand supply network resilience in different network structures.

\section{Results}

The approach described in "Action-based networks" section is used to infer action-based models for each of the real-world SCNs listed in Table 1. Additionally, the action-based framework was also used to synthesize supply networks that reflect the observations of 


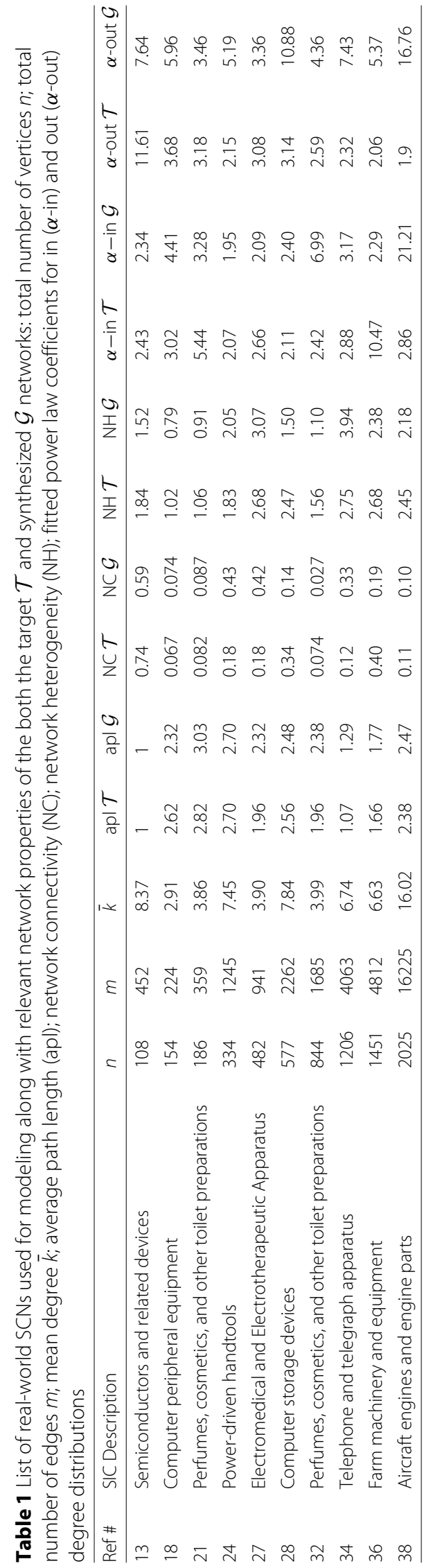


empirical studies, such as power law degree distributions and disassortative mixing. The inferred probabilistic models are also used to draw conclusions about the individual local mechanisms that are primarily responsible for link formation in SCNs. The networks synthesized using the learned models were then subjected to random and targeted disruptions of nodes, and evaluated against two resilience metrics. This provides an indirect yet effective way of analyzing the ability of a network to remain functional under adverse circumstances.

\section{Dataset}

We begin by describing the real-world supply chain dataset that was used in this research. Supply chain data provided by (Willems 2008) and analyzed in (Perera et al. 2017b) has been used to investigate the applicability of the proposed framework on different supply chain networks. The dataset contains 38 multiechelon supply chains used for inventory optimization purposes. The supply chains consist of firms with five different roles, namely, parts (suppliers), manufacturers, transportation, distributors and retailers. Tier information (the dataset used the term relative depth) is also available, and different supply chains have between 2 and 10 tiers. The SCNs described in this paper comprise actual supply chain maps created by either company analysts or consultants. This makes the dataset a perfect test bed for validating the efficiency and effectiveness of supply chain models. 10 among the 38 were selected based on network density and size, and they are listed in Table 1 along with eight relevant SCN properties. Two of the SCNs are also shown in Fig. 2 for visual representation. The SCNs shown in Fig. 2 both posses a tiered structure, but the interconnectedness among various tiers and the number of nodes in each tier is very different in the two networks.

A key limitation of the SCN dataset is the absence of data on geographical locations of individual firms. This information was not provided in the original dataset due to confidentiality reasons. As discussed earlier, geographical location might play a significant role in linking decisions of firms and its unavailability might significantly limit our understanding of various structural features. Furthermore, this empirical study does not

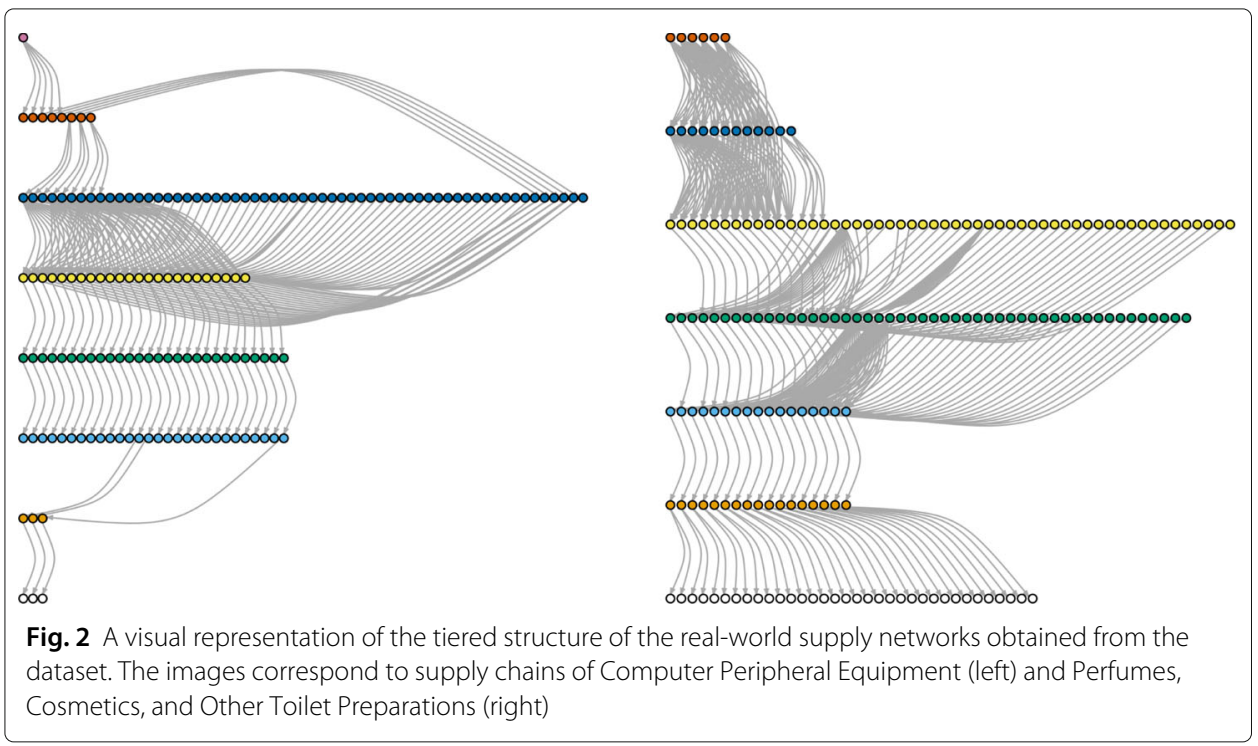


explore the dynamic nature of the SCNs since the dataset does not provide any information pertaining to temporal changes in the SCN topology. Lastly, the dataset does not provide information regarding amount of material flow between connected firms. Although specific production capabilities of firms within each tier are known, no information is available in relation to how much each upstream firm supplies to the downstream firms. Nevertheless, the size of the dataset, both in terms of the number of networks available and in terms of number of nodes in each network, make this a very attractive dataset to study.

\section{Modeling SCNs}

To test the ability of ABNG to replicate distinct global network properties observed in real-world SCNs, the generator was tested using 10 target networks from the dataset described in "Dataset" section. The list of SCNs that were considered for modeling is provided in Table 1 along with eight relevant network level metrics of the target networks and the corresponding networks synthesized using ABNG. Figure 3 presents a summary of the results, featuring heat maps for the 10 target networks. The solution closest to the origin (based on 1-norm) was chosen as the action-based model and used to synthesize 20 networks. The mean dissimilarity values are recorded in the heat maps by comparing the 2-sample Kolmogorov-Smirnov statistic value for betweenness, in-degree and outdegree between the target and synthesized networks (these were used as objectives during the optimization). Additionally, we also provide mean values of absolute deviation of the average path length, network centralization and network heterogeneity between the target and synthesized networks (see (Kim et al. 2015; Perera et al. 2017a) for details and definitions of these properties and their relevance in the context of supply chains).

As seen in Fig. 2, the target networks might impose strict constraints on how nodes in two tiers are connected, for example the green and blue nodes in the supply chain of Computer Peripheral Equipment have a one-to-one mapping. The constraints imposed by such specialized sub-structures might lead to synthesis of disconnected networks using ABNG. Synthesis of SCNs that are not fully connected is

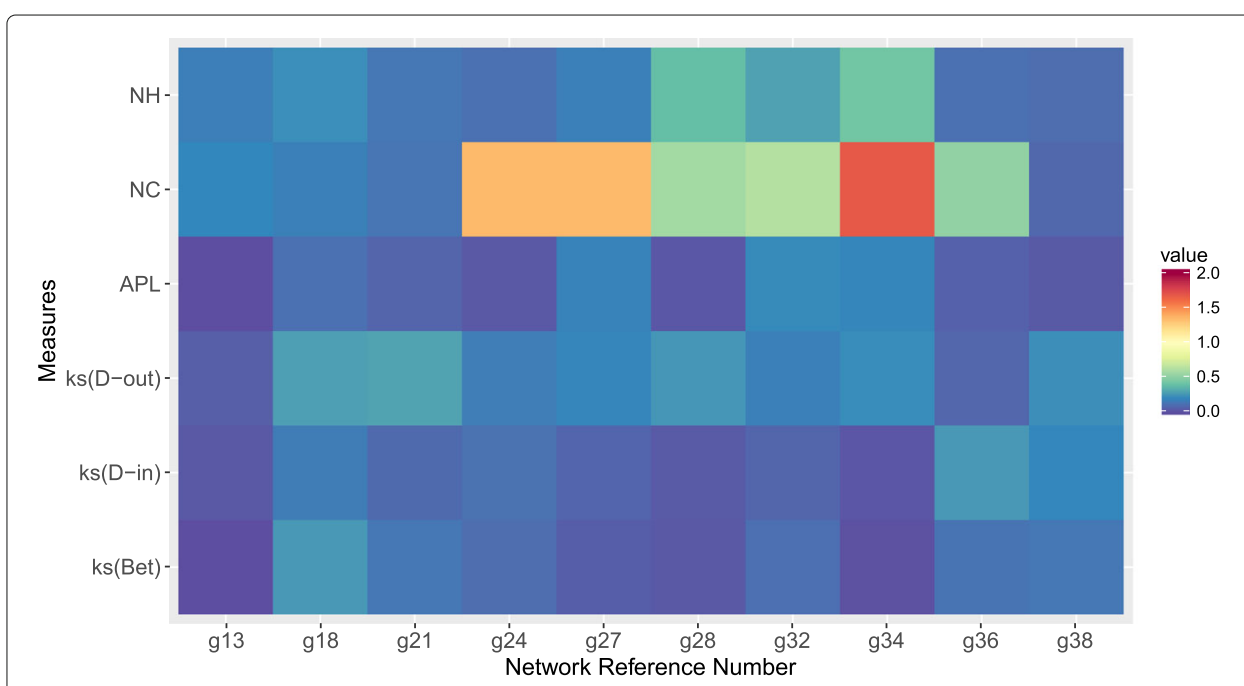

Fig. 3 Results of measures for the 10 SCNs modeled using ABNG. The solution closest to the origin (based on 1 -norm) was chosen as the action-based model 
not a desirable outcome. To deal with this issue, a clean-up phase was devised to ensure every node participating in the supply chain is connected to the synthesized $\mathrm{SCN}$. If the algorithmic procedure described in Algorithm 1 synthesizes a disconnected network, the clean-up phase is initiated to create a connected supply chain by randomly connecting a disconnected node to a node that is already a part of the overall supply chain, while adhering to tier constraints described in "Incorporating tier constraints" section. The networks obtained after the clean-up phase are then subjected to further analysis.

An action matrix corresponding to the solution closest to the origin (based on 1norm) obtained for three of the real-world SCNs is shown in Table 2. This can help the user in making some conclusions about the structure of these networks and the propensity with which each action is used to form links. ABNG was also used to synthesize an artificial SCN with statistical properties observed in most empirical studies, i.e. in and out degree distributions with power law coefficients $\alpha=2$ and disassortative mixing among nodes (see Fig. 4 for a visual of the synthesized network). The action matrices obtained for each of these networks have some similarities, but with subtle differences. It can be seen that most of the SCNs have a corresponding action-based model consisting of nodes belonging to only two or three different node types, i.e. most of the firms use similar local mechanisms to form links. A common observation is that "no action" tends to have high probability. A possible conclusion here is that only a few nodes add edges in a time step, leading to a power law degree distribution in the network. Further, most networks use preferential attachment on in-degree, degree difference and betweenness as dominant mechanisms for forming links. This provides evidence that firms that get more supplies tend to attract more connections, firms tend to link disassortatively and try to connect with nodes in shortest paths. The SCN on computer storage devices is an exception where nodes tend to link based on closeness and out-degree with higher probability. It should be noted that the action based on distances does not get a high probability because the current version uses distance as number of hops in the network. If geographical location is available in the dataset, this action is likely to have much higher probability.

Table 2 The table shows optimized action matrix for a few SCNs

\begin{tabular}{llllllllll}
\hline Network $\downarrow$ | Action $\rightarrow$ & PAOD & PAID & PADD & PAB & PAC & Rand & InvSD & NA & $\mathbb{P}(T=t)$ \\
\hline Perfumes, cosmetics, and & 0.000 & 0.174 & 0.015 & 0.108 & 0.037 & 0.076 & 0.158 & 0.432 & 0.188 \\
Other toilet preparations & 0.007 & 0.249 & 0.179 & 0.074 & 0.000 & 0.008 & 0.000 & 0.483 & 0.812 \\
& 0.077 & 0.032 & 0.009 & 0.373 & 0.026 & 0.000 & 0.000 & 0.483 & 0.508 \\
Power-driven handtools & 0.053 & 0.193 & 0.236 & 0.223 & 0.096 & 0.021 & 0.178 & 0.000 & 0.091 \\
& 0.018 & 0.000 & 0.000 & 0.094 & 0.023 & 0.010 & 0.000 & 0.855 & 0.401 \\
& 0.266 & 0.000 & 0.388 & 0.024 & 0.082 & 0.089 & 0.000 & 0.151 & 0.203 \\
Computer storage devices & 0.226 & 0.017 & 0.143 & 0.116 & 0.167 & 0.017 & 0.314 & 0.000 & 0.186 \\
& 0.205 & 0.000 & 0.208 & 0.169 & 0.229 & 0.123 & 0.066 & 0.000 & 0.611 \\
Artificial SCN & 0.020 & 0.001 & 0.000 & 0.183 & 0.000 & 0.000 & 0.000 & 0.796 & 0.851 \\
& 0.132 & 0.156 & 0.251 & 0.091 & 0.132 & 0.071 & 0.108 & 0.059 & 0.149 \\
\hline
\end{tabular}

The following actions were used: Preferential attachment on: out-degree (PAOD), in-degree (PAID), degree difference (PADD), betweenness (PAB), closeness (PAC); Random selection (Rand); Inverse shortest distance (InvSD); and No action (NA) 


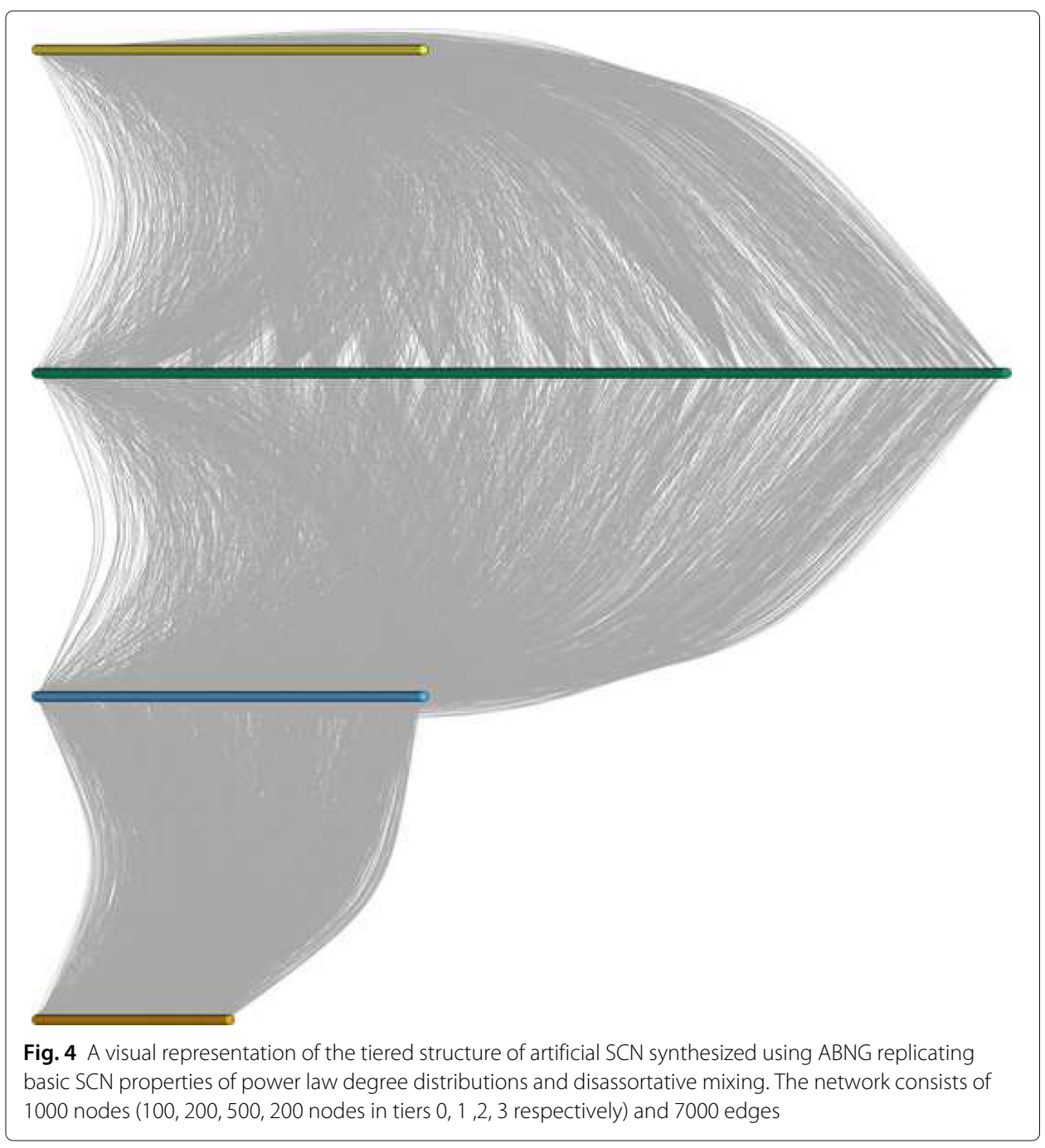

\section{Resilience analysis}

The supply chain literature emphasizes that specific measures are required for evaluating topological resilience of SCNs by incorporating the role of various nodes in the network. Analytical measures of resilience commonly used in the network science literature (Rubinov and Sporns 2010; Costa et al. 2005) are unable to account for node heterogeneity, which is a critical aspect in SCN modeling. For example, (Zhao et al. 2011a, b) point out that in the context of SCNs, the distance between two supply nodes or two demand nodes are not as important as that between a supply and a demand node. To tackle this issue, researchers rely on simulation based metrics to analyze topological resilience through customized metrics. The usual approach consists of simulating random or targeted disruptions by removing nodes from the network. (Perera et al. 2017a) provides an outline of the methodological framework that is typically used for analysis of topological resilience of SCNs. This procedure consists of sequentially repeating the following steps:(i) simulate node removal, and (ii) measure the relevant resilience metrics. This can provide general insights into the topological aspects of $\mathrm{SCN}$ resilience. 
To incorporate the heterogeneous roles of firms for resilience analysis in SCN, the set of nodes $V$ can be divided into supply $\left(V_{S}\right)$ and demand $\left(V_{D}\right)$ nodes. We assume that the final consumers are the demand nodes $\left(V_{D}=T_{0}\right)$, and every other node is a supply node $\left(V_{S}=V \backslash V_{D}\right)$. For a network to be resilient, the most important requirement is to ensure that the demand nodes have access to at least one supply node. Supply availability rate measures the percentage of demand nodes that have access to supply nodes, hence providing an estimate of whether the demand nodes have access to supplies for maintaining normal operations. As shown in Eq. 2, the supply availability rate $S_{A}$ can be calculated as the ratio of number of consumers that still have access to a supplier to the total number of consumers.

$$
S_{A}=\frac{\left|V_{D}^{\prime}\right|}{\left|V_{D}\right|}, \text { where } V_{D}^{\prime}=\left\{v_{i} \in V_{D} \mid \exists v_{j} \in V_{S}: \exists \text { path between } v_{i} \text { and } v_{j}\right\}
$$

The second measure, size of the functional network, corresponds to the number of nodes in the largest connected component that has at least one supply node, thus serving as a measure of supply network connectivity. For calculating the size of the largest functional network, we first need to find the largest connected component that satisfies the required conditions. Let $V_{\text {sub }}$ be the set of nodes in the remaining functional network, then a node in $V_{\text {sub }}$ should satisfy the following requirements:

$$
\text { (i) } \forall v_{i}, v_{j} \in V_{\text {sub }}: \exists \text { path between } v_{i} \text { and } v_{j} \text {, and (ii) } \exists v_{k} \in V_{\text {sub }}: v_{k} \in V_{S}
$$

Once a set of measures for evaluating topological resilience of a network have been decided, the sequential procedure described earlier can be used to perform analysis on a network. In this research, we simulate random disruptions by randomly removing a supply node from the network, and targeted disruptions remove a supply node with the highest total degree (sum of in and out degree). Though we perform degree-based targeted disruptions, variations that use different centrality measures can also be used. Another useful technique to study the impact of removing nodes from a network is to devise a classification scheme for nodes in a SCN with reference to resilience. This can be achieved by altering the procedure outlined in (Savvopoulos and Moschoyiannis 2017) to incorporate $\mathrm{SCN}$ resilience, but is out of scope for this study.

Figures 5, 6, 7, 8 and 9 show the effect of random and targeted removal of nodes from the synthesized and real-world networks on supply availability and size of the functional network under disruptions. The ABNG-synthesized network corresponds to those created by the modeling procedure described in this paper (followed by the clean-up phase, if required). Each blue line corresponds to the average resilience values of 20 synthesized networks, with the highlighted region capturing the networks lying between the 5 th and 95th quantile. It is interesting to note that average resilience of the networks synthesized using our approach are generally comparable to or better than the considered real-world networks under both disruption scenarios. (Perera et al. 2017b) analyzed the SCNs that we use as target networks and concluded that the networks show high structural resilience. This implies that the actions used in this research can be used to make informed decisions leading to design of resilient supply networks as the synthesized networks have resilience comparable to the target networks.

The artificial SCN shown in Fig. 4 was also subjected to resilience analysis, and the results can be seen in Fig. 10. It should be noted that because there were no constraints 


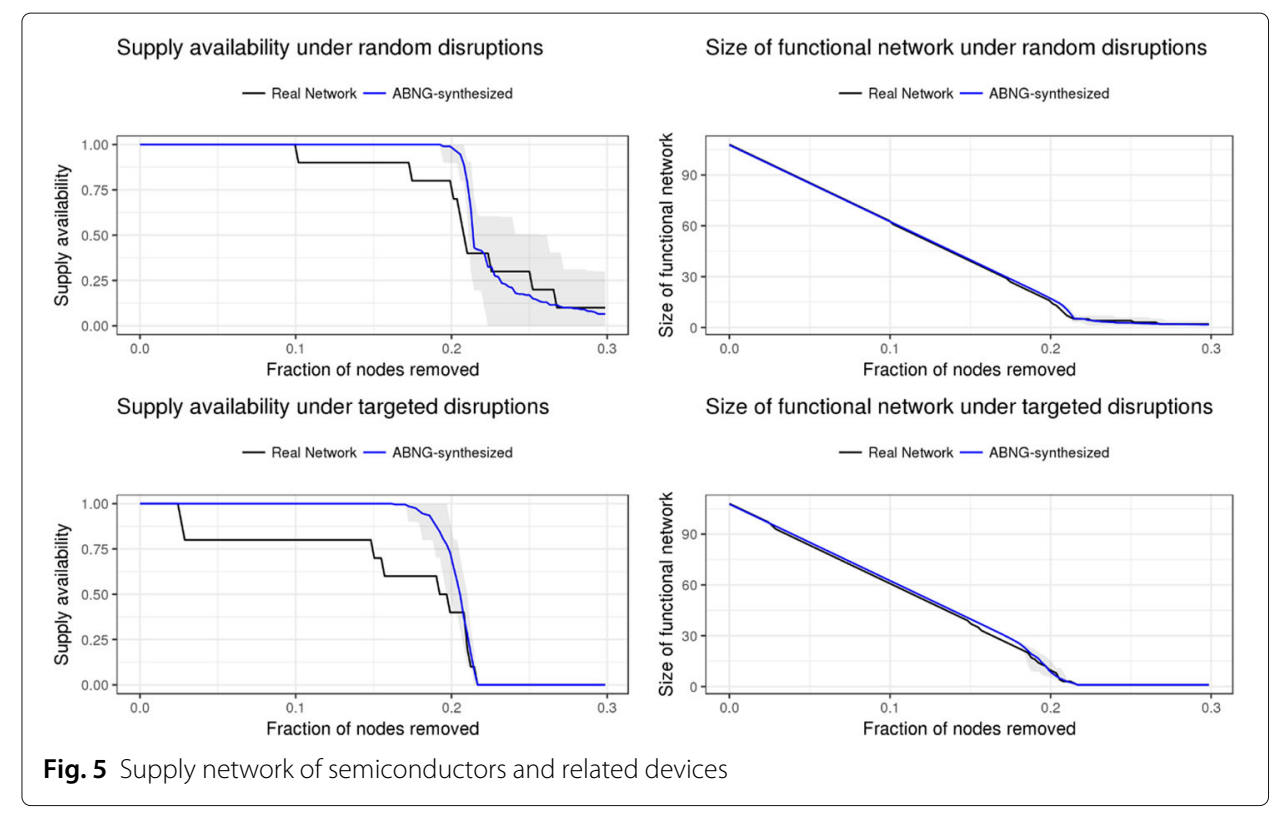

from a target network, the synthesized networks are fully connected. Another important observation is that there is very little variation (small highlighted portion in Fig. 10) because of the absence of real-world constraints on the network structure.

Overall, the proposed model is capable of synthesizing networks that are structurally similar to real-world SCNs only by utilizing a few global network properties in form of objective functions $S(\mathcal{T})$, and also incorporating context-dependent constraints on link formation between firms in different tiers. The ability of the synthesized networks to retain functionality under disruption demonstrates that the micro level linking decisions (actions) of individual firms can be used in a probabilistic manner to synthesize topologically resilient network structures. Using the information from node level actions and

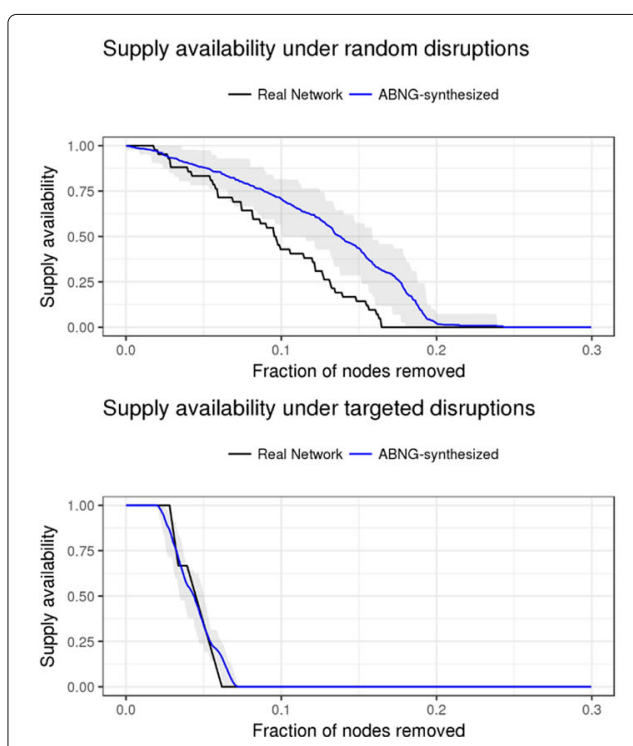

Size of functional network under random disruptions — Real Network — ABNG-synthesized

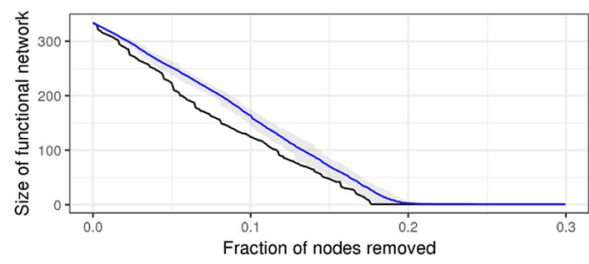

Size of functional network under targeted disruptions

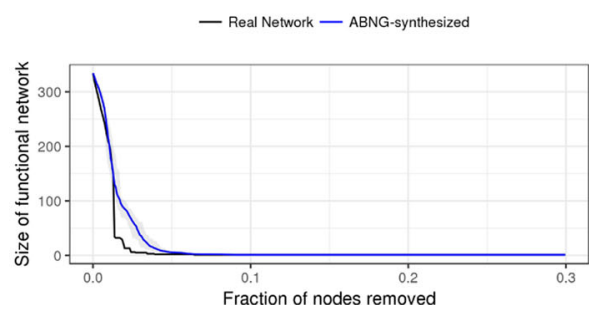

Fig. 6 Supply network of power-driven handtool 


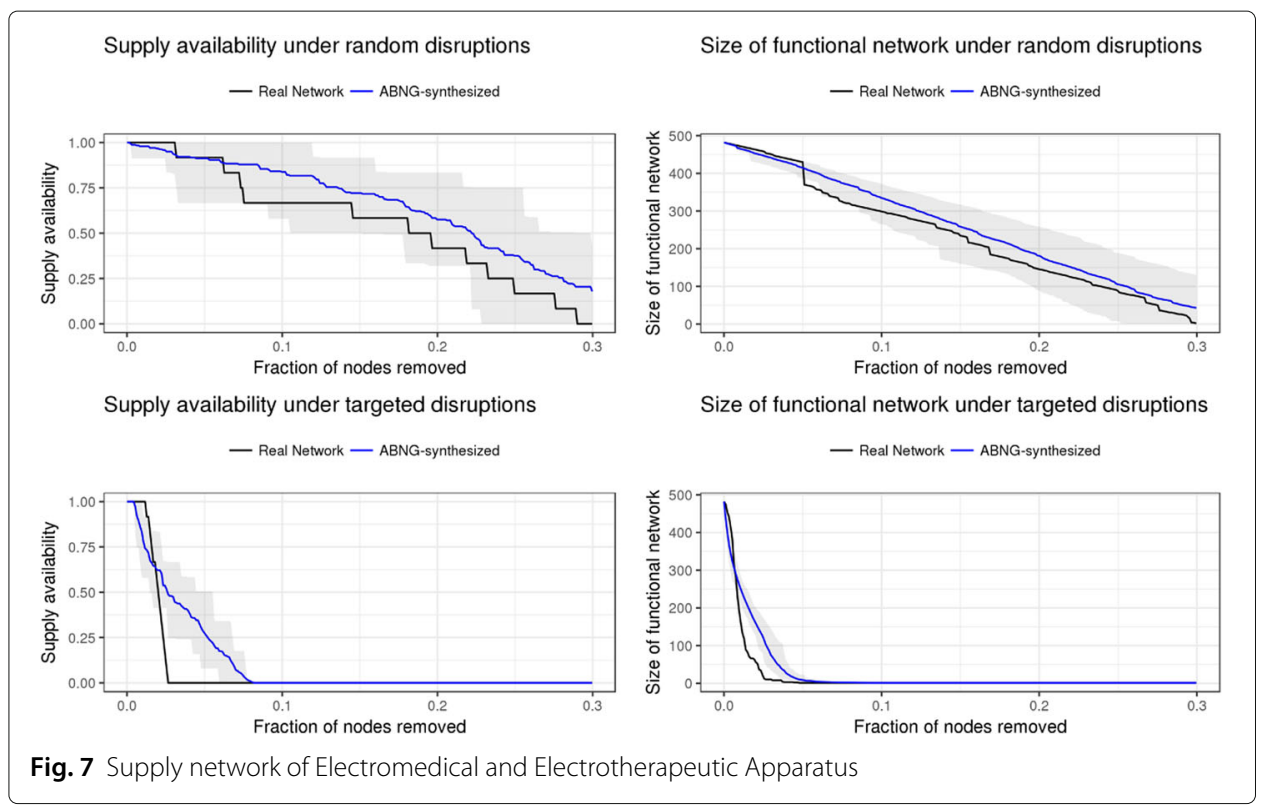

corresponding probabilities can provide network designers with a better understanding of supply chain dynamics, and hence make informed decisions regarding designing systems that can retain functionality under disruptions.

\section{Conclusions and future work}

The objective of this research was to investigate the possibility of using a network model to synthesize resilient supply networks capable of structurally replicating a given realworld supply chain. The framework incorporates essential features of SCNs like node heterogeneity by using tier information and allowing different mechanisms to connect with firms. The results indicate that decisions by firms at the micro level can lead to

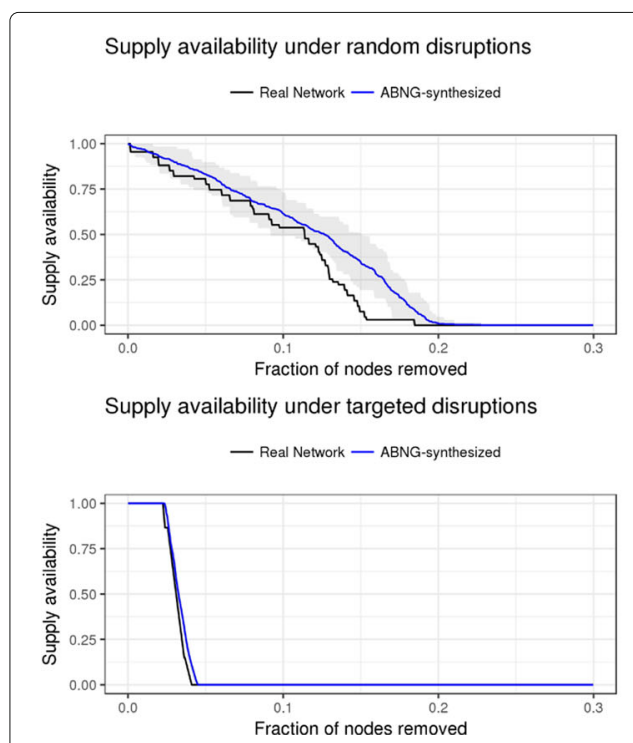

Size of functional network under random disruptions

— Real Network — ABNG-synthesized

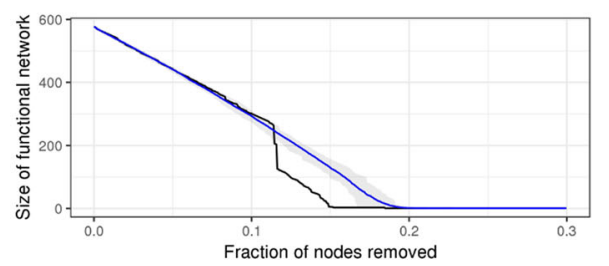

Size of functional network under targeted disruptions

- Real Network - ABNG-synthesized

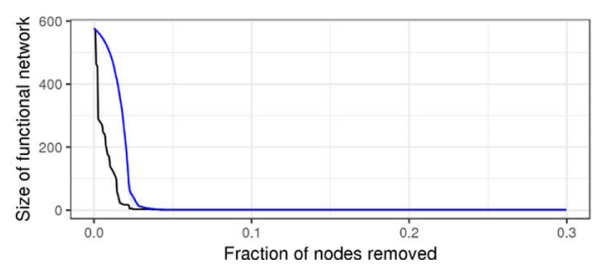

Fig. 8 Supply network of Computer Storage Devices 


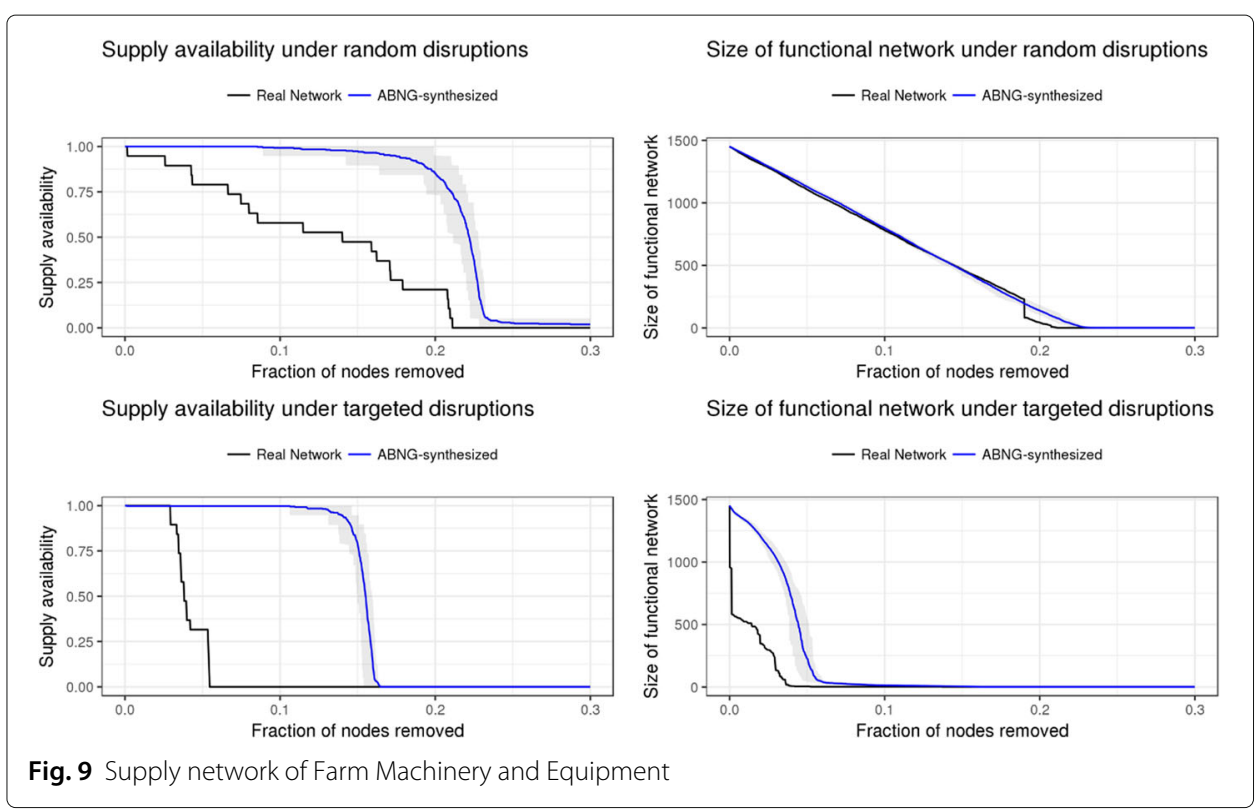

creation of networks that exhibit topological resilience, hence providing insights into network design principles. The framework can be extended to capture dynamics of such networks by adding features such as arrival of new nodes and rewiring of existing edges. Information regarding node demands and incorporating tighter constraints on demand fulfillment can make the model more representative of real-world SCNs, but the unavailability of material flow data might hinder the progress in this direction. Availability of demand data will also lead to synthesis of connected networks by ensuring that individual demands are satisfied. Firm fitness (generally evaluated using profit or loss) is seen as an important driving mechanism for supply chain evolution (Li et al. 2009, 2010) and can be included as an additional action in future research, and also used as a metric for addition
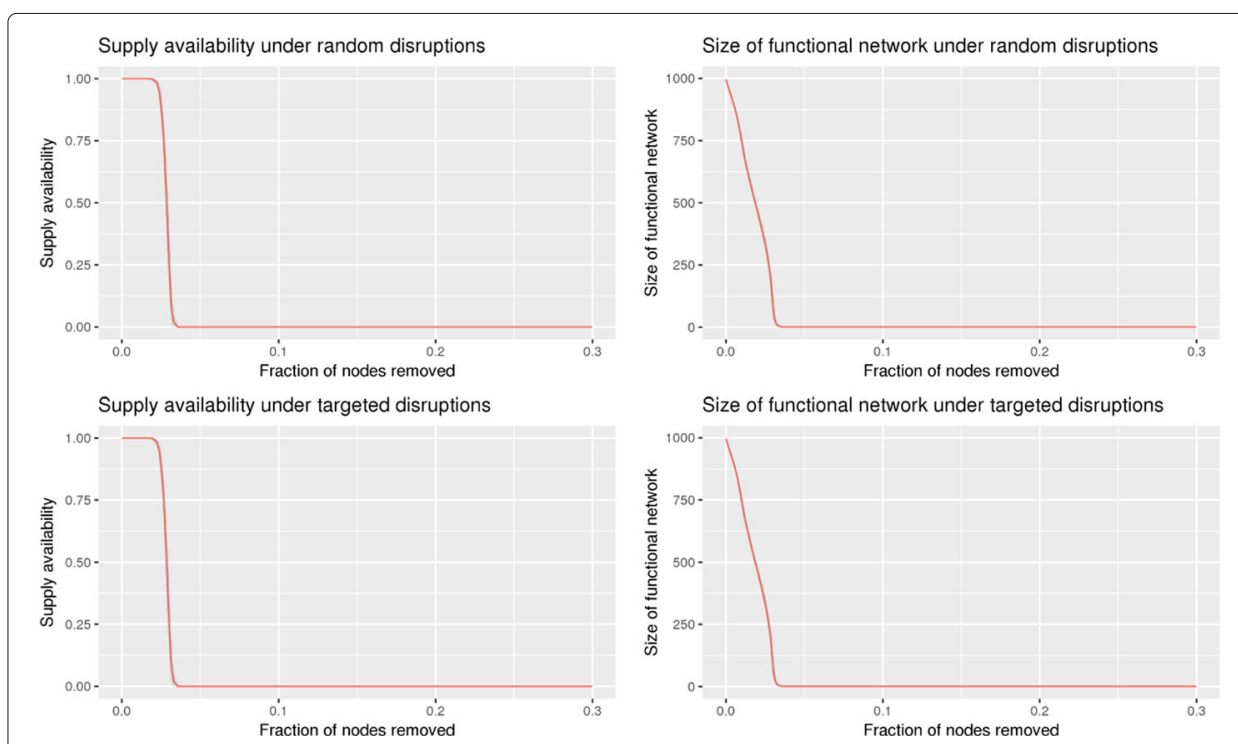

Fig. 10 Artificial supply network 
and removal of firms/nodes in the network. This can effectively capture the dynamics of supply chains and how the evolution of the network effects its resilience.

\section{Endnotes}

${ }^{1}$ This action is currently based on shortest distances in the network. If available, node information about location of firms can also be used.

\section{Acknowledgements}

We would like to thank two anonymous referees for their comments and suggestions.

\section{Funding}

This material is based upon work supported by the National Science Foundation under Grant No. 1549608.

\section{Availability of data and materials}

The datasets used in this article are all publicly available and cited in the references.

\section{Authors' contributions}

VA and MV contributed equally to this work. Both authors read and approved the final manuscript.

\section{Competing interests}

The authors declare that they have no competing interests.

\section{Publisher's Note}

Springer Nature remains neutral with regard to jurisdictional claims in published maps and institutional affiliations.

Received: 18 February 2018 Accepted: 13 June 2018

Published online: 09 July 2018

\section{References}

Alcantara P, Riglietti G, Aguada L (2017) BCI Supply Chain Resilience Report. Tech Rep.1-52

Alderson DL (2009) Catching the 'Network Science' Bug: Insight and Opportunities for the Operations Researchers. Oper Res 56(5):1047-1065

Anderson CJ, Wasserman S, Crouch B (1999) A p* primer: Logit models for social networks. Soc Networks 21(1):37-66

Arora V, Ventresca M (2017) Action-based Modeling of Complex Networks. Sci Rep 7(1):6673

Arora V, Ventresca M (2018) Action-Based Model for Topologically Resilient Supply Networks. In: Studies in Computational Intelligence, vol. 689. Springer International Publishing, Cham. pp 658-669

Barabási AL, Albert R (1999) Emergence of scaling in random networks. Science 286(October):509-512

Barroso AP, Machado VH, Carvalho H, Cruz Machado V (2015) Quantifying the Supply Chain Resilience. In: Applications of Contemporary Management Approaches in Supply Chains. InTech, Rijeka. pp 13-32

Barthélemy M (2011) Spatial networks. Phys Rep 499(1-3):1-101

Bell M, Perera S, Piraveenan M, Bliemer M, Latty T, Reid C (2017) Network growth models: A behavioural basis for attachment proportional to fitness. Sci Rep 7(October 2016):42431

Bellamy MA, Basole RC (2013) Network analysis of supply chain systems: A systematic review and future research. Syst Eng 16(2):235-249

Borgatti SP, Li X (2009) On Social Network Analysis In A Supply Chain Context. J Supply Chain Manag 45(2):5-22 Brintrup A, Ledwoch A, Barros J (2016) Topological robustness of the global automotive industry. Logist Res 9(1):1

Brintrup A, Wang Y, Tiwari A (2015) Supply Networks as Complex Systems: A Network-Science-Based Characterization. IEEE Syst J PP(99):1-12

Choi TY, Dooley KJ, Rungtusanatham M (2001) Supply networks and complex adaptive systems: control versus emergence. J Oper Manag 19(3):351-366

Costa LF, Rodrigues FA, Travieso G, Boas PRV (2005) Characterization of complex networks: A survey of measurements. Adv Phys 56(1):167-242

Czyzak P, Jaszkiewicz A (1998) Pareto Simulated Annealing-A Metaheuristic Technique for Multiple-Objective Combinatorial Optimization. J Multi-Criteria Decis Anal 7(1):34-47

Erdös P, Rényi A (1959) On random graphs. Publ Math Debr 6:290-297

Fay D, Moore AW, Brown K, Filosi M, Jurman G (2014) Graph metrics as summary statistics for Approximate Bayesian Computation with application to network model parameter estimation. J Complex Netw 3:009

Fienberg SE (2012) A Brief History of Statistical Models for Network Analysis and Open Challenges. J Comput Graph Stat 21(4):825-839

Goldenberg A, Zheng AX, Fienberg SE, Airoldi EM (2010) A Survey of Statistical Network Models. Found Trends Mach Learn 2(2):129-233

Gao J, Barzel B, Barabási AL (2016) Universal resilience patterns in complex networks. Nature 530(7590):307-312

Harrison KR, Ventresca M, Ombuki-Berman BM (2016) A meta-analysis of centrality measures for comparing and generating complex network models. J Comput Sci 17:205-215

Hearnshaw EJS, Wilson MMJ (2013) A complex network approach to supply chain network theory. Int J Oper Prod Manag 33(4):442-469 
Jain V, Wadhwa S, Deshmukh SG (2009) Select supplier-related issues in modelling a dynamic supply chain: Potential, challenges and direction for future research. Int J Prod Res 47(11):3013-3039

Keqiang WKW, Zhaofeng ZZZ, Dongchuan SDS (2008) Structure Analysis of Supply Chain Networks Based on Complex Network Theory. 2008 Fourth International Conference on Semantics, Knowledge and Grid:493-494

Kim Y, Chen YS, Linderman K (2015) Supply network disruption and resilience: A network structural perspective. J Oper Manag 33-34:43-59

Kim Y, Choi TY, Yan T, Dooley K (2011) Structural investigation of supply networks: A social network analysis approach. J Oper Manag 29(3):194-211

Klibi W, Martel A, Guitouni A (2010) The design of robust value-creating supply chain networks: A critical review. Eur J Oper Res 203(2):283-293

Li G, Gu YG, Song ZH (2013) Evolution of cooperation on heterogeneous supply networks. Int J Prod Res 51(13):3894-3902

Li G, Ji P, Sun LY, Lee WB (2009) Modeling and simulation of supply network evolution based on complex adaptive system and fitness landscape. Comput Ind Eng 56(3):839-853

Li G, Yang H, Sun L, Ji P, Feng L (2010) The evolutionary complexity of complex adaptive supply networks: A simulation and case study. Int J Prod Econ 124(2):310-330

Lomi A, Pattison P (2006) Manufacturing Relations: An Empirical Study of the Organization of Production Across Multiple Networks. Organ Sci 17(3):313-332

Mari SI, Lee YH, Memon MS, Soo Park Y, Kim M (2015) Adaptivity of Complex Network Topologies for Designing Resilient Supply Chain Networks. Int J Ind Eng Theory, Appl Pract 22:102-116

Nair A, Vidal JM (2011) Supply network topology and robustness against disruptions - an investigation using multi-agent model. Int J Prod Res 49(5):1391-1404

Newman MEJ (2003) The Structure and Function of Complex Networks. SIAM Rev 45(2):167-256

Pathak SD, Dilts DM, Biswas G (2007) On the evolutionary dynamics of supply network topologies. IEEE Trans Eng Manag 54(4):662-672

Pathak SD, Dilts DM, Mahadevan S (2009) Investigating population and topological evolution in a complex adaptive supply network. J Supply Chain Manag 45(3):54-67

Perera SS, Bell MGH, Bliemer MCJ (2015) Modelling Supply Chains as Complex Networks for Investigating Resilience : An Improved Methodological Framework. In: Australasian Transport Research Forum (ATRF). pp 1-16

Perera S, Bell MGH, Bliemer MCJ (2017a) Network science approach to modelling the topology and robustness of supply chain networks: a review and perspective. Appl Netw Sci 2(1):33

Perera S, Perera HN, Kasthurirathna D (2017b) Structural characteristics of complex supply chain networks. In: 2017 Moratuwa Engineering Research Conference (MERCon). IEEE, Piscataway. pp 135-140

Perera S, Perera HN, Kasthurirathna D (2017c) Value chain approach for modelling resilience of tiered supply chain networks. In: Engineering Research Conference (MERCon). IEEE, Moratuwa. pp 159-164

Pržulj N (2007) Biological network comparison using graphlet degree distribution. Bioinformatics 23(2):177-183

Robins G, Pattison P, Kalish Y, Lusher D (2007) An introduction to exponential random graph ( $p^{*}$ ) models for social networks. Soc Networks 29(2):173-191

Rubinov M, Sporns O (2010) Complex network measures of brain connectivity: Uses and interpretations. Neurolmage 52(3):1059-1069

Savvopoulos S, Moschoyiannis S (2017) Impact of removing nodes on the controllability of complex networks. In: COMPLEX NETWORKS 2017: The 6th International Conference on Complex Networks \& Their Applications. pp 361-363

Song C, Havlin S, Makse Ha (2006) Origins of fractality in the growth of complex networks_nature. Nat Phys 2(April):275-281

Strauss D (1986) On a General Class of Models for Interaction. SIAM Rev 28(4):513-527

Surana A, Kumara S, Greaves M, Raghavan UN (2005) Supply-chain networks: a complex adaptive systems perspective. Int J Prod Res 43(20):4235-4265

Thadakamalla HP, Raghavan UN, Kumara S, Albert A (2004) Survivability of Multiagent-Based Supply Networks: A Topological Perspective. IEEE Intell Syst 19(5):24-31

Wang W, Street WN, et al (2015) Topological resilience analysis of supply networks under random disruptions and targeted attacks. In: Advances in Social Networks Analysis and Mining (ASONAM), 2015 IEEE/ACM International Conference on. IEEE. pp 250-257

Wasserman S, Pattison P (1996) Logit models and logistic regressions for social networks. Psychometrika 60:401-425

Watts DJ, Strogatz SH (1998) Collective dynamics of 'small-world' networks,. Nature 393(6684):440-2

Wen L, Guo M, Wang L (2012) Statistic Characteristics Analysis of Directed Supply Chain Complex Network. Int J Advancements Comput Technol 4(21):84-91

Willems SP (2008) Data Set —Real-World Multiechelon Supply Chains Used for Inventory Optimization. Manuf Serv Oper Manag 10(1):19-23

Xuan Q, Du F, Li Y, Wu T-j (2011) A Framework to Model the Topological Structure of Supply Networks. IEEE Trans Autom Sci Eng 8(2):442-446

Yaveroğlu ÖN, Malod-Dognin N, Davis D, Levnajic Z, Janjic V, Karapandza R, Stojmirovic A, Pržulj N (2015) Revealing the Hidden Language of Complex Networks. Sci Rep 4(1):4547

Zhao K, Kumar A, Yen J (2011a) Achieving High Robustness in Supply Distribution Networks by Rewiring. IEEE Trans Eng Manag 58(2):347-362

Zhao K, Kumar A, Harrison TP, Yen J (2011 b) Analyzing the Resilience of Complex Supply Network Topologies Against Random and Targeted Disruptions. IEEE Syst J 5(1):28-39

Zheng B, Wu H, Kuang L, Qin J, Du W, Wang J, Li D (2014) A simple model clarifies the complicated relationships of complex networks. Sci Rep 4:6197 